\title{
Subtenon Injection of Autologous Platelet-Rich Plasma in Retinitis Pigmentosa: Is It a New Therapeutic Option?
}

\author{
Neslihan Sinim Kahraman, Ayse Oner* \\ Ophthalmology, Department of Ophthalmology, Acıbadem Kayseri Hospital, Kayseri, Turkey \\ Email: neslihansinim@gmail.com, ${ }^{*}$ aoner@erciyes.edu.tr, ${ }^{*}$ ayseozoner@gmail.com
}

How to cite this paper: Kahraman, N.S. and Oner, A. (2020) Subtenon Injection of Autologous Platelet-Rich Plasma in Retinitis Pigmentosa: Is It a New Therapeutic Option? Open Journal of Ophthalmology, $10,77-88$

https://doi.org/10.4236/ojoph.2020.101010

Received: November 24, 2019

Accepted: January 7, 2020

Published: January 10, 2020

Copyright $\odot 2020$ by author(s) and Scientific Research Publishing Inc. This work is licensed under the Creative Commons Attribution International License (CC BY 4.0).

http://creativecommons.org/licenses/by/4.0/ (c) (i) Open Access

\begin{abstract}
Purpose: Growth factors (GFs) and neurotrophins can decelerate retinal degeneration. This study aimed to investigate the safety, efficacy and durability of subtenon injections of autologous platelet-rich plasma (aPRP) which is a rich source of GFs in retinitis pigmentosa (RP) patients. Methods: 154 eyes of 77 RP patients with various degrees of narrowed visual field were investigated in this study. Each patient received three injections with 4-week intervals and followed for at least 12 months. The examinations and the tests were obtained before the injection, 1 month after the third injection and every 3 months during the study. The primary aim was to evaluate the effects of aPRP on visual acuity (VA) and visual functions, the second aim was to estimate the duration of the therapy effect and the need for additional aPRP injection. Results: Median age of the $77 \mathrm{RP}$ patients was $35.2 \pm 13.9$ years. All of the eyes received 3 monthly bilateral subtenon aPRP injections. Of these patients 26 received an additional one, 12 received additional two and 1 patient received additional 3 injections with 3-month interval. There were no significant ocular or systemic side effects. The mean baseline VA was $0.22 \pm 0.18$ Snellen lines. It improved to $0.31 \pm 0.19$ following three aPRP injections, which was statistically significant. At the end of the study period, the mean VA was 0.27 \pm 0.22 Snellen lines. Conclusion: The subtenon injection of aPRP might be an effective therapy and might have a positive influence in the preservation of visual functions and visual acuity of RP patients.
\end{abstract}

\section{Keywords}

Retinitis Pigmentosa, Growth Factors, Platelet-Rich Plasma, Visual Functions 


\section{Introduction}

Retinitis pigmentosa (RP) is a hereditary retinal dystrophy of photoreceptors and an important cause of severe vision impairment. It might cause potential blindness by age 40 - 50. The presence of nyctalopia, visual field construction from periphery to the fovea, bone spicule pigmentation in the retina and a reduction in electroretinograms (ERGs) are seen as the findings of the disease. Even if the mechanisms of retinal cell death can be different, photoreceptor apoptosis is the final outcome in the disease course [1]. Finding an efficient treatment would help the patients' quality of life affected by visual deterioration. There is presently no curative treatment for RP, but there are some studies about rescue strategies [2].

Retina pigment epithelium (RPE) protects the remaining part of the retina against toxic and oxidative damage and secrete growth factors (GFs). The deficiency of GFs in photoreceptors may be one of the predisposing factors for the degeneration of these cells [3].

In the eye, basic fibroblast GF (bFGF), neural GF (NGF), ciliary neurotrophic factor (CNTF), brain-derived neurotrophic factor (BDNF) and glial cell line-derived neurotrophic factor (GDNF) are known for their neurotrophic effects. Application of these factors may block the apoptotic cascade of retinal cells and may be a promising treatment option in the future. Studies in the treatment of retinal degenerations have largely focused to prevent or decrease the progression of these diseases [3].

Recently, blood-derived products have been mostly used in ophthalmology as a GF source. These products can increase the healing process and accelerate the regeneration of various tissues by supplying GFs and other active biomolecules from the blood. Topical autologous serum was widely-used and found to be effective in the treatment of various ocular surface diseases [4] [5] [6] [7] [8]. Platelet rich plasma (PRP) contains higher concentrations of essential GFs and cell adhesion molecules than autologous serum. Experimental studies showed that GFs and neurotrophins can significantly decelerate retinal degeneration and cell death [3] [9].

There are a few clinical studies in the literature demonstrating the efficacy of subtenon injection of autologous PRP (aPRP) in retinal degenerative and ischemic diseases [10] [11]. This study aimed to evaluate a long term effect of subtenon aPRP on visual acuity and visual field in patients with RP.

\section{Methods}

This clinical study included 154 eyes of $77 \mathrm{RP}$ patients attending to the ophthalmology clinic of our hospital between January 2018 and January 2019. The study followed the tenets of the Declaration of Helsinki. The patients were informed about the details, aims and the course of the study. Written informed consent was obtained from each subject before any of the study procedures or examinations were performed. The diagnosis of RP was established depending on the clinical history, ophthalmological findings, visual field (VF) test, optical cohorence tomography (OCT) and electroretinography (ERG) test results of the patients. 
The inclusion criteria were as follows: 1) a clinical diagnosis of RP confirmed by clinical history, fundus appearance, VF, OCT and ERG; 2) subjects older than 18 years of age; 3 ) subjects who are able to do a reliable VF evaluation; 4) subjects who have at least 1 year-follow-up results.

Patients with previous ocular surgery other than cataract extraction, ocular media opacities that would make the image quality insufficient for ocular imaging or effect the test results, coexisting ocular disease (e.g., retinal pathology other than RP, glaucoma, uveitis, strabismus, nystagmus), any other systemic disease (e.g., diabetes, neurological diseases, hypertension) that would have an impact on the results were excluded from the study.

A single experienced vitreoretinal surgeon (AO) performed all the surgical procedures and ophthalmic evaluations. Baseline ophthalmic evaluation of the patients included best corrected visual acuity (BCVA), applanation tonometry, slit lamp biomicroscopy, color fundus photography, OCT, VF and ERG. Visual acuity was evaluated by using a Snellen chart at a distance of $3 \mathrm{~m}$. OCT was performed using the Optovue (Optovue Inc, USA) with a standardized scanning protocol. VF examination was performed by (the Threshold 30-2 Humphrey VF by HFAII750 device (Carl Zeiss Meditec AG, Germany) ERG (ERG-Vision monitor, Monpack 3, Metrovision, France) readings were recorded from each eye according to the International Society for Clinical Electrophysiology of Vision (ISCEV) guideline [12]. All tests were performed with the same instrument by the same technician.

Preparation of autologous PRP (aPRP): Previous studies reported different methods to obtain PRP. Whole blood is collected in acid/citrate/dextrose and centrifuged. In all procedures the final PRP product usually has a platelet count which is $4-5$ times higher than baseline [13] [14]. In our study, peripheral blood of $15 \mathrm{ml}$ from RP patients was collected using blood collection tubes (BD Vacutainer ii advance) and centrifugation was done with $2500 \mathrm{rpm}$ for 15 minutes at room temperature within a blood collection period of 10 minutes. After this centrifugation process the plasma was separated from the other part of blood components. Three layers including red blood cells at the bottom, aPRP in the middle layer and platelet poor plasma (PPP) in the top layer formed in the tubes. Middle layer (which mainly contained platelets) was drawn by syringe. Subtenon injections were performed immediately after the preparation of aPRP. After topical anesthesia aPRP solution was injected from inferotemporal quadrant into the subtenon space of each eye. Each patient received three injections with 4 -week intervals between each injection. The patients were examined every 3 or 4 months for at least 10 months after the third injection; thus, the total duration of the study for each patient was 1 year. The examinations and the tests of the patients including OCT and VF were obtained before the injection, 1 month after the third injection and at every visit until the end of the first year. ERG test was done before the injections and at the end of the first year. Additional aPRP injections were done if a detoriation in the VF and visual functions was detected. 
In this clinical study, the primary aim was to evaluate the effects of aPRP on BCVA, VF, and ERG; the second aim was to evalute the duration of the therapy effect and the need for additional aPRP injection.

Analyses were carried out with SPSS for Windows (v22; IBM Corp.; Armonk, NY, USA). A p value of 0.05 or less was considered as statistically significant; repeated ANOVA was used to analyze the BCVA, OCT, VF and ERG parameters. The results were defined as mean values with their standard deviation (SD).

\section{Results}

This study included 154 eyes of $77 \mathrm{RP}$ patients with a median age of $35.2 \pm 13.9$ years. The demographic characteristics of the patients were given in Table 1. All of the eyes received 3 monthly bilateral subtenon aPRP injections. Of these patients, 26 (33.7\%) received additional 1 injection (16 patients received additional injection after 3 months, 10 of them received injection after 6 months), 12 (15.5\%) patients received additional 2 injections with 3 months interval and 1 (1.3\%) patient received 3 additional injections with 3 months interval. During the first year 39 (50.5\%) of the patients needed additional injections. There were no serious ocular or systemic side effects related to aPRP preparations and subtenon injections. The mean BCVA of the 154 studied eyes before treatment was $0.22 \pm 0.18$ Snellen lines. It improved to $0.31 \pm 0.19$ following three monthly aPRP applications which were statistically significant $(\mathrm{p}<0.05)$. At the end of the follow-up period the mean BCVA decreased to $0.27 \pm 0.22$, the result was not statistically significant when compared to baseline. Visual acuity measurements of eyes during the study are shown in Table 2.

Table 1. The demographic characteristics of participants.

\begin{tabular}{cc}
\hline Number of patients & 77 \\
Number of eyes & 154 \\
Age (Years) & $35.2 \pm 13.9$ \\
Mean \pm SD & $(18-63)$ \\
Gender (F/M) & $37 / 40$ \\
\hline
\end{tabular}

Table 2. Comparison of BCVA, CMT and VF measurements of the patients during the follow-up examinations.

\begin{tabular}{cccc}
\hline & BCVA (Snellen line) & CMT $(\mu \mathrm{m})$ & Visual field MD $(\mathrm{dB})$ \\
\hline Baseline value before aPRP & $0.22 \pm 0.18$. & $79.81 \pm 30.20$ & $-29.06 \pm 6.12$ \\
Second value after $3^{\text {rd }}$ aPRP & $0.31 \pm 0.19$ & $82.34 \pm 28.58 \mu \mathrm{m}$ & $-27.93 \pm 7.11$ \\
Final value after 6 $6^{\text {th }}$ month & $0.27 \pm 0.22$, & $84.6 \pm 26.43$ & $-23.59 \pm 10.71$ \\
P Value & $\mathrm{P}<0.05^{*}$ & $\mathrm{p}>0.05^{* *}$ & $<0.05^{* * *}$
\end{tabular}

BCVA, best-corrected visual acuity; aPRP, autologous platelet-rich plasma; CMT, central macular thickness; MD, Mean deviation; $\mathrm{dB}$, Decibel, ${ }^{\star}$ The difference between baseline BCVA and second BCVA was statistically significant however there was not a difference between baseline BCVA and final BCVA. ${ }^{*}$ There was not a statistically significant difference between macular thickness measurements. ${ }^{* *}$ There was a statistically significant difference between all measurements. 
Visual field values were obtained in all eyes. Statistically significant VF improvement was detected after 3 monthly injections when compared to pre-injection values and the improvement continued in most of the patients during the study period $(\mathrm{p}<0.05)$ (Table 2, Figure 1 and Figure 2).

When compared with pretreatment values we found no statistically significant difference in the $\mathrm{b}$ wave amplitudes and latencies of rod response, maximal combined response and cone response after aPRP injections and at the final examination $(\mathrm{p}>0.05)$ (Table 3$)$. There were also no changes in the macular thickness or anatomy by OCT at any time point (Table 2).
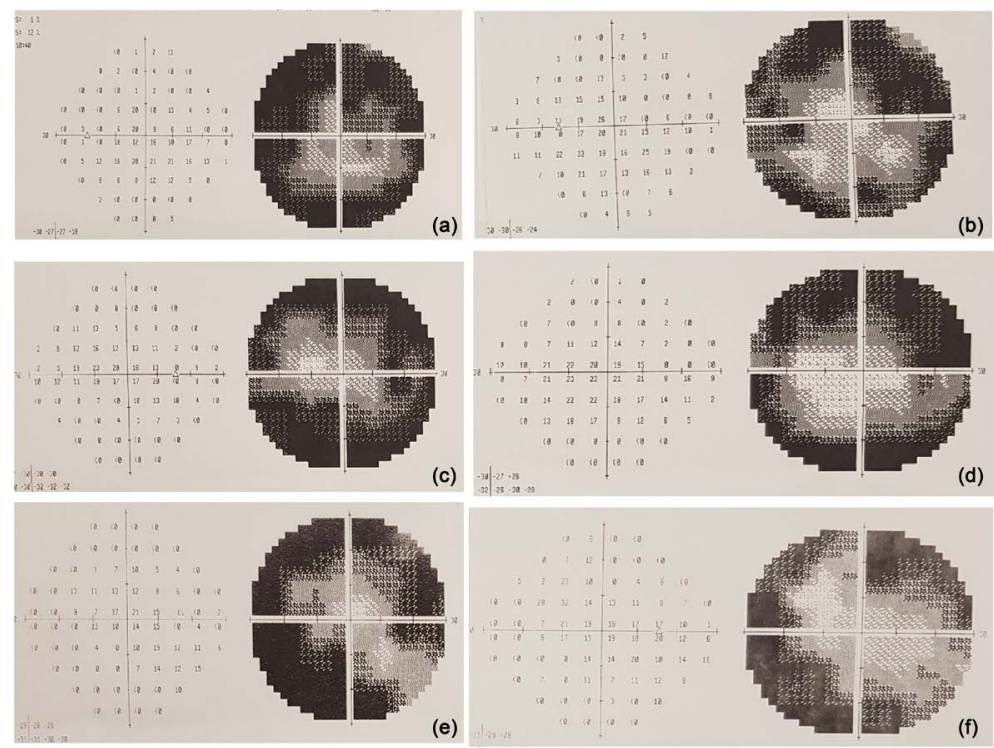

Figure 1. Visual field changes after aPRP injections. ((a), (c), (e)) Before aPRP application; ((b), (d), (f)) After $3^{\text {rd }}$ aPRP application: Note the improvement in VF test. These patients did not receive any additional injections.
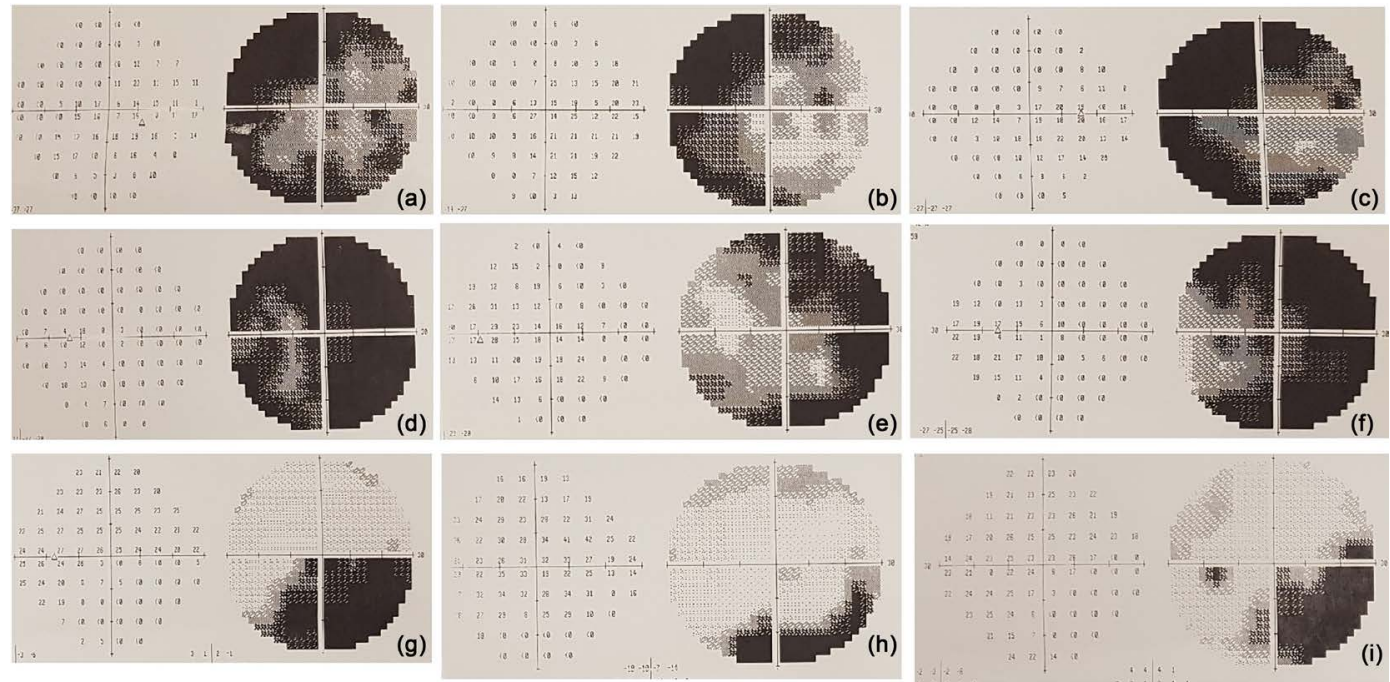

Figure 2. Visual field changes after aPRP injections. ((a), (d), (g)) Before aPRP application, ((b), (e), (h)) After $3^{\text {rd }}$ aPRP application, ((c), (f), (i)) 3 months after $3^{\text {rd }}$ aPRP application. These patients received additional injections because of the detoriation in the VF test. 
Table 3. The results of electrophysiological tests before and after treatment.

(amp: $\mu \mathrm{V}$ lat: $\mathrm{ms}$ )

\begin{tabular}{cccc}
\hline ERG Tests & Before Treatment & After Treatment & P value \\
\hline Rod response b wave amplitude & $43.00 \pm 28.76$ & $45.50 \pm 24.01$ & $>0.05$ \\
Rod response b wave latency & $81.49 \pm 13.44$ & $87.26 \pm 8.17$ & $>0.05$ \\
Maximal combined response b wave amplitude & $58.76 \pm 56.50$ & $60.67 \pm 73.98$ & $>0.05$ \\
Maximal combined response b wave latency & $83.88 \pm 18.40$ & $84.29 \pm 13.28$ & $>0.05$ \\
Cone response b wave amplitude & $26.51 \pm 18.15$ & $30.16 \pm 33.18$ & $>0.05$ \\
Cone response b wave latency & $41.86 \pm 3.03$ & $49.49 \pm 71.87$ & $>0.05$ \\
\hline
\end{tabular}

\section{Discussion}

Despite the great developments in the genetic and molecular biological understanding of retinal degenerations in recent years, the exact pathways of retinal cell death have not yet been fully understood. Estimated causes of photoreceptor death are thought to be toxic and oxidative damage, deficiency of growth factors and metabolic impairment. Retinal cells can enter into a sleep mode which is called dormant state under unfavorable situations. This phase is necessary to rescue the cell from death. However, the way in which cells enter into and recover from this sleep mode is not fully understood yet. Recent gene therapy studies showed that these dormant cells may respond to external rescue cues such as gene therapy or other pharmacological treatments [15].

It is known that apoptosis is one of the major reasons of photoreceptor cell death. The deprivation of GFs leads to the induction of apoptosis mechanism. At the beginning, retinal cells decelerate their metabolic activities. If the GF insufficiency persists, the apopitosis process of the photoreceptors takes place. This raises the possibility that GFs may play an inhibitory role in the apoptosis process and GF levels may be essential in maintaining viability of cells undergoing apoptosis [16].

Neurotrophic factors are the GFs which have an important role in the development and normal activity of neurons. Thus, applications of these neurotrophic factors are thought to be an alternative for the protection of retinal photoreceptors from degeneration [16].

Various factors such as CNTF, BDNF, GDNF and pigment epithelium-derived factor (PEDF) have been investigated in experimental studies and demonstrated to rescue the retinal structure [17]. Because photoreceptors are thought to be a part of central nerve system, neurotrophic factors might be beneficial for retinas of RP patients to preserve retinal cells [18].

NGF is one of the most important neuroprotective factor which plays an important role in the pathogenesis of retinal cell damage [19]. The amount of NGF and NGF-receptors are found to be decreased in retinas of animals with RP, and external NGF application preserved photoreceptors from degeneration [20] [21]. It is confirmed by the studies that application of NGF accelerated the expression 
of multiple GFs including BDNF, FGF, transforming growth factor beta (TGF- $\beta$ ), VEGF and neuropeptide-Y (NPY). NGF may also stimulate the expression of VEGF and enhance outer retinal oxygenation by vasodilatation [21]. Pharmacodynamic studies have shown that topically administered NGF on the cornea can reach the retina of the eye. In a clinical study, the usage of NGF eye drops to the subjects with advanced glaucoma induced improvement of visual acuity [22] [23]. The neuroprotective effect of NGF was also confirmed by other studies. Intravitreal injection of recombinant human NGF (rhNGF) inhibited apoptosis and increased retinal cell survival in an experimental RP model [24]. A recent report of a clinical trial showed that NGF eye drops had no systemic or ocular adverse effect in RP patients. Some of these patients demonstrated an increase in visual acuity, a temporary enlargement of the VF and an improvement in macular focal ERG findings. This study suggested that the application of NGF eye-drop is safe and efficient in at least some of the RP patients [25].

Another approach of neuroprotection has been tested with CNTF, which is widely used in experimental studies and decelerate retinal degeneration. In experimental RP models, intravitreal administration of this factor protected photoreceptors from degeneration. After successful results of animal studies, safety studies in humans are established [26] [27] [28] [29]. In a phase 1 clinical trial of $\mathrm{RP}$, intraocular implant of CNFT has been demonstrated to be safe and some of the patients experienced increases in visual acuity [29]. Another study of CNTF implant had found that fellow eyes of the patients had greater retinal cell loss than CNTF-implanted eyes in 2 years period. And this protection of retinal cells in CNTF-implanted eyes caused a thickening of outer retinal layers on OCT [30]. The initial 12-month analysis of a phase 2 study in RP patients has demonstrated a dose-dependent thickening of the retina on OCT imaging [31]. However long term follow-up showed no benefit of CNTF implant regarding the visual acuity and visual field [32].

GDNF is of a particular interest due to its known broad spectrum of targets, including dopaminergic neurons, motor neurons of spinal cord, as well as retinal ganglion cells and photoreceptors of the eye. It has been shown to enhance the development and survival of a wide variety of neurons, including ganglion cells and photoreceptors [33] [34]. It is also reported in an experimental study that GDNF accelerates the threshold for apoptosis in photoreceptors [35].

Since 1998, platelet-derived products have been widely used in regenerative medicine [9] [36]. These products included many bioactive molecules such as epidermal growth factor (EGF), PDGF, VEGF, insulin-like growth factor (IGF-1), TGF- $\beta$ and bFGF which took part in tissue repair and wound healing process [22] [36] [37].

The studies in ophthalmology about platelet derived products are mostly about ocular surface diseases like dry eye syndrome or corneal ulcers [4] [5] [6] [7] [8]. There are only two clinical studies about retinal diseases which used aPRP as a source of autologous GFs. In a recent study by Arslan et al. the aim of 
aPRP injections was thought to promote the survival of retinal cells, to increase neuroprotection, to inhibit photoreceptor apoptosis by using GFs and to reactivate dormant photoreceptor cells. The study included 71 eyes of $48 \mathrm{RP}$ patients who received three subtenon aPRP injections with 3 -week intervals and they were followed for three more weeks after the third injection. This study suggested that the patients experienced improvements in VF, mfERG, and microperimetry results after aPRP injections however, there were no significant changes in visual acuity. They concluded that aPRP administration can increase the amount of neurotrophic GFs in the microenvironment around the retina and the diffusion of these factors through the choroid can reactivate the photoreceptors that are in dormant phase. They have reported no systemic or ocular side effects regarding the procedure. They also suggested that further researchs are needed to determine the duration of viability and the optimal frequency of aPRP reinjections [10].

Another recent clinical study by Ozmert et al. [11] investigated the efficacy of retinal electromagnetic stimulation and subtenon aPRP in the treatment of deep retinal capillary ischemia and included 28 eyes of 17 patients. They divided the eyes into three groups: group 1 received electromagnetic stimulation alone; group 2 received electromagnetic stimulation and subtenon aPRP; group 3 was a control group and had no intervention. Electromagnetic stimulation was performed in ten sessions of in groups 1 and 2. In addition, group 2 received three subtenon aPRP during the study. The deep retinal capillary density and visual acuity changes were investigated before and after treatment at the first month. Although both treatment modalities improved the mean deep retinal capillary density significantly, the results of the combined group (group 2) were significantly superior to the monotherapy group (group 1). The researchers conclude that subtenon fresh aPRP injection together with electromagnetic stimulation may be effective in the treatment of local ischemia of the retina and prevent permanent retinal damage.

Similar to the previous studies, we found improvements in VA and VF evaluations of our patients. However the improvement in VA after three aPRP injections detoriated in nearly half of the patients after 3 months and the patients needed additional injections. The effect of the treatment seems to be preserved for at least 3 months.

Our study has several limitations. First, the patient group was not homogeneous in respect to VA and VF tests. This may explain the different durability of the therapy. Second, a study of genetics and its correlation with various functional tests is not included in this study. Genetic analysis may guide us to understand the response to the aPRP treatment in different genetic mutations.

\section{Conclusion}

$\mathrm{RP}$ is the most common hereditary retinal disease with no proven effective therapies. This study shows encouraging results after subtenon injection of aPRP 
with no systemic and ocular side effects. The subtenon injection of aPRP has a beneficial effect on VA and VF. This positive effect may be transient in some patients and additional injections may be necessary during the follow-up. Future randomized controlled trials including genetical analysis are needed to understand the effect of the treatment in different genetic mutations.

\section{Ethics Approval and Consent to Participate}

All procedures performed in this study were in accordance with the ethical standards of the institutional research committee and with the 1964 Helsinki declaration and its later amendments or comparable ethical standards. All patients were instructed about the objectives and methodology of the study and gave written informed consent to participate.

\section{Consent for Publication}

All patients gave written informed consent for publication of images.

\section{Funding}

There is no funding received for this study.

The manuscript has not been published elsewhere previously and it is not under consideration in any other journal.

All authors have contributed significantly and are in agreement with the content of the manuscript.

\section{Conflicts of Interest}

The authors declare no conflicts of interest regarding the publication of this paper.

\section{References}

[1] Hartong, D.T., Berson, E.L. and Dryja, T.P. (2006) Retinitis Pigmentosa. The Lancet, 368, 1795-1809. https://doi.org/10.1016/S0140-6736(06)69740-7

[2] Oner, A. (2018) Stem Cell Treatment in Retinal Diseases: Recent Developments. Turkish Journal of Ophthalmology, 48, 33-38. https://doi.org/10.4274/tjo.89972

[3] Kolomeyer, A.M. and Zarbin, M.A. (2014) Trophic Factors in the Pathogenesis and Therapy for Retinal Degenerative Diseases. Survey of Ophthalmology, 59, 134-165. https://doi.org/10.1016/j.survophthal.2013.09.004

[4] Tsubota, K., Goto, E., Shimmura, S. and Shimazaki, J. (1999) Treatment of Persistent Corneal Epithelial Defect by Autologous Serum Application. Ophthalmology, 106, 1984-1989. https://doi.org/10.1016/S0161-6420(99)90412-8

[5] Matsumoto, Y., Dogru, M., Goto, E., et al. (2004) Autologous Serum Application in the Treatment of Neurotrophic Keratopathy. Ophthalmology, 111, 1115-1120. https://doi.org/10.1016/j.ophtha.2003.10.019

[6] Goto, E., Shimmura, S., Shimazaki, J. and Tsubota, K. (2001) Treatment of Superior Limbic Keratoconjunctivitis by Application of Autologous Serum. Cornea, 20, 807-810. https://doi.org/10.1097/00003226-200111000-00006 
[7] Noda-Tsuruya, T., Asano-Kato, N., Toda, I. and Tsubota, K. (2006) Autologous Serum Eye Drops for Dry Eye after LASIK. Journal of Refractive Surgery, 22, 61-66. https://doi.org/10.3928/1081-597X-20060101-13

[8] Na, K.S. and Kim, M.S. (2012) Allogeneic Serum Eye Drops for the Treatment of Dry Eye Patients with Chronic Graft-versus-Host Disease. Journal of Ocular Pharmacology and Therapeutics, 28, 479-483. https://doi.org/10.1089/jop.2012.0002

[9] De Pascale, M.R., Sommese, L., Casamassimi, A. and Napoli, C. (2015) Platelet Derivatives in Regenerative Medicine: An Update. Transfusion Medicine Reviews, 29, 52-61. https://doi.org/10.1016/j.tmrv.2014.11.001

[10] Arslan, U., Özmert, E., Demirel, S., Örnek, F. and Şermet, F. (2018) Effects of Subtenon-Injected Autologous Platelet-Rich Plasma on Visual Functions in Eyes with Retinitis Pigmentosa: Preliminary Clinical Results. Graefe's Archive for Clinical and Experimental Ophthalmology, 256, 893-908. https://doi.org/10.1007/s00417-018-3953-5

[11] Ozmert, E. and Arslan, U. (2019) Management of Deep Retinal Capillary Ischemia by Electromagnetic Stimulation and Platelet-Rich Plasma: Preliminary Clinical Results. Advances in Therapy, 36, 2273-2286.

https://doi.org/10.1007/s12325-019-01040-2

[12] Marmor, M.F., Fulton, A.B., Holder, G.E., Miyake, Y., Brigell, M. and Bach, M. (2009) ISCEV Standard for Full-Field Clinical Electroretinography (2008 Update) (for the International Society for Clinical Electrophysiology of Vision). Documenta Ophthalmologica, 118, 69-77. https://doi.org/10.1007/s10633-008-9155-4

[13] Mazzucco, L., Balbo, V., Cattana, E. and Borzini, P. (2008) Platelet Rich Plasma and Platelet Gel Preparation Using Platelet. Vox Sanguinis, 94, 202-208. https://doi.org/10.1111/j.1423-0410.2007.01027.x

[14] Jo, C.H., Roh, Y.H., Kim, J.E., Shin, S. and Yoon, K.S. (2013) Optimizing Platelet-Rich Plasma Gel Formation by Varying Time and Gravitational Forces during Centrifugation. Journal of Oral Implantology, 39, 525-532. https://doi.org/10.1563/AAID-JOI-D-10-00155

[15] Koenekoop, R.K. (2011) Why Some Photoreceptors Die, While Others Remain Dormant: Lessons from RPE65 and LRAT Associated Retinal Dystrophies. Ophthalmic Genetics, 32, 126-128. https://doi.org/10.3109/13816810.2010.544361

[16] Collins, M.K., Perkins, G.R., Rodriguez-Tarduchy, G., Nieto, M.A. and López-Rivas, A. (1994) Growth Factors as Survival Factors: Regulation of Apoptosis. BioEssays, 16, 133-138. https://doi.org/10.1002/bies.950160210

[17] LaVail, M.M., Yasumura, D., Matthes, M.T., et al. (1998) Protection of Mouse Photoreceptors by Survival Factors in Retinal Degenerations. Investigative Ophthalmology \& Visual Science, 39, 592-602.

[18] Weissmiller, A.M. and Wu, C. (2012) Current Advances in Using Neurotrophic Factors to Treat Neurodegenerative Disorders. Translational Neurodegeneration, 1, 14. https://doi.org/10.1186/2047-9158-1-14

[19] Micera, A., Lambiase, A., Aloe, L., Bonini, S., Levi-Schaffer, F. and Bonini, S. (2004) Nerve Growth Factor Involvement in the Visual System: Implications in Allergic and Neurodegenerative Diseases. Cytokine \& Growth Factor Reviews, 15, 411-417. https://doi.org/10.1016/j.cytogfr.2004.09.003

[20] Lambiase, A. and Aloe, L. (1996) Nerve Growth Factor Delays Retinal Degeneration in $\mathrm{C} 3 \mathrm{H}$ Mice. Graefe's Archive for Clinical and Experimental Ophthalmology, 1, 96-100. https://doi.org/10.1007/BF02343055 
[21] Lenzi, L., Coassin, M., Lambiase, A., Bonini, S., Amendola, T. and Aloe, L. (2005) Effect of Exogenous Administration of Nerve Growth Factor in the Retina of Rats with Inherited Retinitis Pigmentosa. Vision Research, 45, 1491-1500. https://doi.org/10.1016/j.visres.2004.12.020

[22] Lambiase, A., Aloe, L., Centofanti, M., et al. (2009) Experimental and Clinical Evidence of Neuroprotection by Nerve Growth Factor Eye Drops: Implications for Glaucoma. Proceedings of the National Academy of Sciences of the United States of America, 106, 13469-13474. https://doi.org/10.1073/pnas.0906678106

[23] Lambiase, A., Mantelli, F., Sacchetti, M., Rossi, S., Aloe, L. and Bonini, S. (2011) Clinical Applications of NGF in Ocular Diseases. Archives Italiennes de Biologie, 149, 283-292.

[24] Sachetti, M., Mantelli, F., Rocco, M.L., et al. (2017) Recombinant Human Nerve Growth Factor Treatment Promotes Photoreceptor Survival in the Retinas of Rats with Retinitis Pigmentosa. Current Eye Research, 42, 1064-1068.

https://doi.org/10.1080/02713683.2017.1279634

[25] Benedetto, F., Giancarlo, I., Antonio, C., et al. (2016) NGF Eye-Drops Topical Administration in Patients with Retinitis Pigmentosa, a Pilot Study. Journal of Translational Medicine, 9, 14-18. https://doi.org/10.1186/s12967-015-0750-3

[26] Wen, R., Tao, W., Li, Y. and Sieving, P.A. (2012) CNTF and Retina. Progress in Retinal and Eye Research, 31, 136-151. https://doi.org/10.1016/j.preteyeres.2011.11.005

[27] Cayouette, M., Behn, D., Sendtner, M., Lachapelle, P. and Gravel, C. (1998) Intraocular Gene Transfer of Ciliary Neurotrophic Factor Prevents Death and Increases Responsiveness of Rod Photoreceptors in the Retinal Degeneration Slow Mouse. Journal of Neuroscience, 18, 9282-9293. https://doi.org/10.1523/JNEUROSCI.18-22-09282.1998

[28] Tao, W., Wen, R., Goddard, M.B., et al. (2002) Encapsulated Cell-Based Delivery of CNTF Reduces Photoreceptor Degeneration in Animal Models of Retinitis Pigmentosa. Investigative Ophthalmology \& Visual Science, 43, 3292-3298.

[29] Sieving, P.A., Caruso, R.C., Tao, W., et al. (2006) Ciliary Neurotrophic Factor (CNTF) for Human Retinal Degeneration: Phase I Trial of CNTF Delivered by Encapsulated Cell Intraocular Implants. Proceedings of the National Academy of Sciences of the United States of America, 103, 3896-3901.

https://doi.org/10.1073/pnas.0600236103

[30] Talcott, K.E., Ratnam, K., Sundquist, S.M., et al. (2011) Longitudinal Study of Cone Photoreceptors during Retinal Degeneration and in Response to Ciliary Neurotrophic Factor Treatment. Investigative Ophthalmology \& Visual Science, 52, 2219-2226. https://doi.org/10.1167/iovs.10-6479

[31] Birch, D.G., Weleber, R.G., Duncan, J.L., Jaffe, G.J. and Tao, W. (2013) Ciliary Neurotrophic Factor Retinitis Pigmentosa Study Groups. Randomized Trial of Ciliary Neurotrophic Factor Delivered by Encapsulated Cell Intraocular Implants for Retinitis Pigmentosa. American Journal of Ophthalmology, 156, 283-292. https://doi.org/10.1016/j.ajo.2013.03.021

[32] Birch, D.G., Bennett, L.D., Duncan, J.L., Weleber, R.G. and Pennesi, M.E. (2016) Long-Term Follow-Up of Patients with Retinitis Pigmentosa (RP) Receiving Intraocular Ciliary Neurotrophic Factor Implants. American Journal of Ophthalmology, 170, 10-14. https://doi.org/10.1016/j.ajo.2016.07.013

[33] Kyhn, M.V., Klassen, H., Johansson, U.E., et al. (2009) Delayed Administration of Glial Cell Line-Derived Neurotrophic Factor (GDNF) Protects Retinal Ganglion 
Cells in a Pig Model of Acute Retinal Ischemia. Experimental Eye Research, 89, 1012-1020. https://doi.org/10.1016/j.exer.2009.08.014

[34] Politi, L.E., Rotstein, N.P. and Carri, N.G. (2001) Effect of GDNF on Neuroblast Proliferation and Photoreceptor Survival: Additive Protection with Docosahexaenoic Acid. Investigative Ophthalmology \& Visual Science, 42, 3008-3015.

[35] Ohnaka, M., Miki, K., Gong, Y.Y., et al. (2012) Long-Term Expression of Glial Cell Line-Derived Neurotrophic Factor Slows, But Does Not Stop Retinal Degeneration in a Model of Retinitis Pigmentosa. Journal of Neurochemistry, 122, 1047-1053. https://doi.org/10.1111/j.1471-4159.2012.07842.x

[36] Alio, J.L., Arnalich-Montiel, F. and Rodriguez, A.E. (2012) The Role of "Eye Platelet Rich Plasma" (E-PRP) for Wound Healing in Ophthalmology. Current Pharmaceutical Biotechnology, 13, 1257-1265.

https://doi.org/10.2174/138920112800624355

[37] Anitua, E., Andia, I., Ardanza, B., Nurden, P. and Nurden, A.T. (2004) Autologous Platelets as a Source of Proteins for Healing and Tissue Regeneration. Thrombosis and Haemostasis, 91, 4-15. https://doi.org/10.1160/TH03-07-0440 